\title{
Direct Puncture Tunnel Method for Establishing the Retroperitoneal Cavity: Anatomic Confirmation and Clinical Experience
}

\author{
Jianhua Lan, MD, ${ }^{1,2}$ Banghua Liao, MD, Jianzhong Ai, MD, Liang Zhou, MD, and Kunjie Wang, MD ${ }^{1}$
}

\begin{abstract}
Purpose: The feasibility and safety of the direct puncture tunnel method to establish the retroperitoneal cavity was assessed by CT and clinical experience.

Materials and Methods: Ten patients who underwent retroperitoneoscopic procedure were scanned by CT in the lateral decubitus position. The distance between the lumbar fascia and psoas major muscle (L-P distance) on the puncture line was measured. The angle between the puncture line and the posterior colon margin (P-C angle) was also measured. In total, 292 patients who underwent retroperitoneoscopic procedure were used to establish the retroperitoneal cavity using the direct puncture tunnel method, and complications in these patients were evaluated.

Results: The average L-P distance was $25.0 \mathrm{~mm}$ (left side) and $25.5 \mathrm{~mm}$ (right side) in the lateral decubitus position. The average P-C angle was $26.8^{\circ}$ (left side) and $29.7^{\circ}$ (right side). The retroperitoneal cavity was well established in all 292 patients, and no visceral or blood vessel injury occurred.

Conclusions: CT scans in the lateral recumbent position indicate that there are no visceral and large blood vessels on the puncture path. The scans also provide a window for inserting the first trocar blindly into the retroperitoneum. A high success rate and low complication rate were observed clinically for the direct puncture tunnel method. We consider the direct puncture tunnel method to be a simple, effective, and safe way to establish the retroperitoneal cavity.
\end{abstract}

Keywords: adrenal gland, kidney, retroperitoneal access, retroperitoneal space, retroperitoneoscopy

\section{Introduction}

$\mathbf{I}$ N 1973, WitTMOSER ${ }^{1}$ first used a minimally invasive endoscopic technique to access the retroperitoneum while performing lumbar sympathectomy after blunt dissection with a telescope and pneumatic dissection with carbon dioxide. Both Fitzpatrick and Wickham ${ }^{2}$ and Kerbl et al. ${ }^{3}$ pioneered minimally invasive procedures of the urinary system in the upper retroperitoneum, inventing the retroperitoneoscopic ureterolithotomy and retroperitoneoscopic nephrectomy, respectively. However, it was difficult to perform the retroperitoneoscopic procedure because of its inability to effectively create a space within the dense retroperitoneal fat. In 1992, the atraumatic balloon dissection technique was developed by Gaur, ${ }^{4}$ which expanded the scope of therapeutic retroperitoneoscopy applications. Then, retroperitoneoscopic access to the upper urinary tract was also reconsidered..$^{5,6}$ Retroperitoneoscopic operations are now used globally.

Access for retroperitoneoscopy is different from that for transperitoneal laparoscopy, although the surgical technique for the latter approach is well established. ${ }^{7}$ Many techniques for establishing the retroperitoneal cavity have been proposed, such as the use of a balloon catheter, balloon trocar system, and finger dissection. However, these techniques have shortcomings such as air leakage, balloon rupture, bleeding, peritoneal rupture, and necessitating sutures or expensive materials. No single method has been shown to be optimal, especially for obese patients. ${ }^{8}$ Some authors have proposed the direct insufflation method with a 14-gauge Veress needle to create a pneumoretroperitoneum. However, their subsequent operations were still complex. In this study, we propose a novel simple method for establishing the

\footnotetext{
${ }^{1}$ Department and Institute of Urology, West China Hospital, Sichuan University, Chengdu, China.

${ }^{2}$ Department of Urology, People's Hospital of Guang'an City, Guang'an, China.

A video demonstrating this technique is available online.
} 
retroperitoneal space and safety was assessed by a computed tomography (CT) scan in the lateral decubitus position.

\section{Materials and Methods}

\section{CT study}

In total, 10 of 292 patients who used direct puncture tunnel method for establishing the retroperitoneal cavity were included in this $\mathrm{CT}$ research. There were four men and six women. The average age of the patients was 52 years (range, 21-73). The average body mass index was 22.4 (range, 1928). The reasons for CT scan examination were kidney cysts in four patients, ureteral calculus in three patients, adrenal tumors in two patients, and renal cancer in one patient. A skin mark was made $2 \mathrm{~cm}$ above the iliac crest along the posterior axillary line, which is the first puncture point of our procedures. Two CT scans were obtained while the patients were lying in the left and right decubitus positions. An imaginary line was plotted in these CT films to predict the penetrating route of the first trocar. The distance between the lumbar fascia and psoas major muscle (L-P distance) on the puncture line was measured. The angle between the puncture point and the posterior colon margin ( $\mathrm{P}-\mathrm{C}$ angle) was also measured. The L-P distance and P-C angle are illustrated in the CT image in Fig 1.

\section{Establishing procedure of retroperitoneal cavity}

The study was approved by the Ethics Committee of the People's Hospital of Guang'an City. All patients provided informed consent before participation. One 5-mm and two 10-mm laparoscopic ports were used for each operation (Fig. 2A). After administration of cuffed endotracheal anesthesia and Foley catheter placement, patients were secured in a lateral decubitus position. Then, a 1-cm transverse skin incision was made at the midaxillary line and a point $2 \mathrm{~cm}$ above the upper iliac crest edge. A 10-mm disposable puncture trocar was vertically inserted into the retroperito-
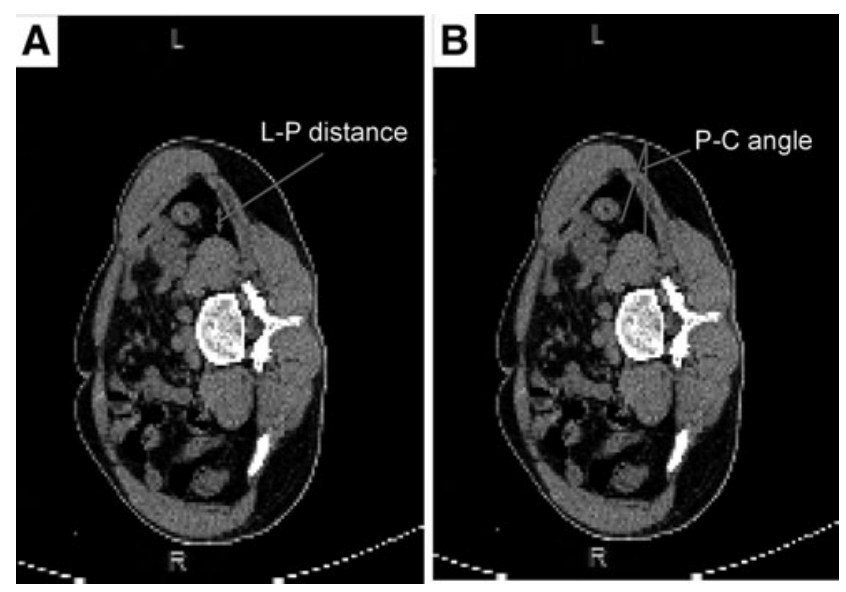

FIG. 1. Computed tomography scans show the anatomical landmarks in the decubitus positions. The distances and angles were measured from an imaginary line and angle, and they were defined as follows: (A) L-P distance was the distance between lumbar fascia and the psoas major muscle on the first puncture line. (B) P-C angle was the angle between the puncture line and the posterior colon margin. neal cavity. After resistance through the lumbodorsal fascia was clearly felt, the obturator was pulled out and carbon dioxide was insufflated at $1 \mathrm{~L} /$ minute (Fig. 2B). A laparoscope was placed into the trocar sheath to assess for organ injury and to confirm the appropriate puncture depth under monitor (N-90X0568-G, STORZ; Germany).

After laparoscope insertion and carbon dioxide diffusion in the retroperitoneum, the laparoscopic tail was depressed, allowing the avascular space anterior to the psoas major muscle to be found quicker. Furthermore, the laparoscope body can be pushed by tunnel under the monitor toward the direction of the costal ridge angle (Fig. 2C). After the retroperitoneal space was clearly displayed at the costal ridge angle, the second trocar was penetrated into the retroperitoneal cavity between the lateral border of the paraspinal muscles and the 12th rib (Fig. 2D).

The second port placement site was similar to that in retroperitoneoscopic procedures on the adrenal gland, kidney, and upper ureter. After the second trocar was inserted, a blunt tipped dissector (e.g., attraction rod) was placed into this trocar sheath. The retroperitoneal fat and peritoneum were passively pressed under the monitor using the blunt tipped dissector to enlarge the retroperitoneal cavity (Fig. 2E). If the vessels and fiber cords from the thoracolumbar fascia were encountered, an ultrasonic knife or electrocoagulation hook was used to prevent bleeding.

After enough retroperitoneal space was acquired, the third trocar was inserted into the retroperitoneal cavity under the monitor at the costal margin of the anterior axillary line, and the retroperitoneal space was well established (Fig. 2F). All three incisions were matched with the puncture trocar; hence, suturing was not required to prevent carbon dioxide leakage. The details of these processes are given in a Supplementary Video S1.

\section{Results}

The results of the CT scan are given in Table 1. Individual variations were observed among these distances and angles; however, the average L-P distance was $25.0 \mathrm{~mm}$ in the left decubitus and $25.5 \mathrm{~mm}$ in the right decubitus; the average angle was $26.8^{\circ}$ in the left decubitus and $29.7^{\circ}$ in the right decubitus.

From December 2017 to August 2019, 292 retroperitoneoscopic procedures were successfully performed using the direct puncture tunnel method. The characteristics of the patients are given in Table 2. In total, 176 cases of the left retroperitoneal cavity and 116 cases of the right retroperitoneal cavity were well established. All 30 patients who underwent laparoscopic radical nephrectomy were for renal tumors. In patients undergoing laparoscopic adrenalectomy, 6 patients were for pheochromocytoma and 50 patients for adrenal adenoma. Laparoscopic nonfunctional nephrectomy was indicated in patients for end-stage hydronephrosis as a result of ureteral calculi obstruction in 30 patients and ureteropelvic junction obstruction in 5 patients. Laparoscopic nephroureterectomy was indicated in patients for carcinoma of the renal pelvis in four patients and ureteral carcinoma in six patients. Laparoscopic ureterolithotomy was completed for a large upper ureteral calculus in 22 patients. Laparoscopic renal cyst decortication was carried out for cysts $>4 \mathrm{~cm}$ with compressive symptoms in 139 patients. All adult 

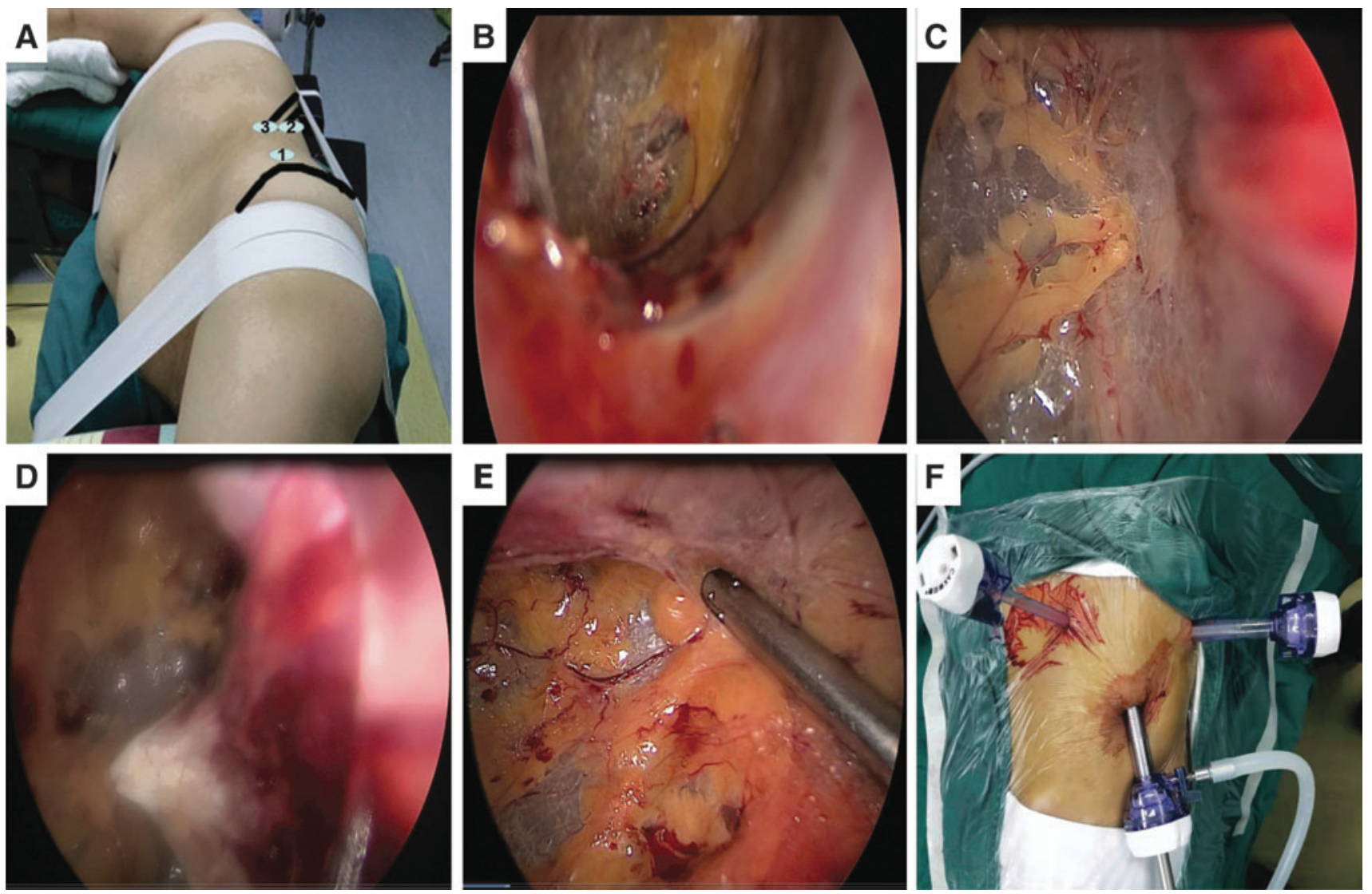

FIG. 2. The process of the direct puncture tunnel method for establishing the retroperitoneal cavity. (A) The patient in the left lateral position with three trocar locations. The first port was inserted with a 10-mm trocar and the laparoscope was placed here. The second port was also inserted with a 10-mm trocar and a blunt-tipped dissector was placed here. Last, the third port was inserted with a 5-mm trocar. (B) The laparoscope was inserted into the first trocar to assess for visceral or blood vessel injury. (C) The laparoscope head was advanced toward the rib angle in the avascular space in front of the psoas major muscle under visualization. (D) A $10-\mathrm{mm}$ trocar was inserted in the second hole (patient in the left lateral position). (E) The retroperitoneal fat and peritoneum were passively pressed under the monitor using the blunt tipped dissector to enlarge the retroperitoneal space. (F) The third trocar was inserted at the costal margin of the anterior axillary line.

Table 1. Anatomic Distances and Angles

Measured by Computed Tomography SCAN (Right Decubitus/Left Decubitus)

\begin{tabular}{lcc}
\hline Patient no. & $L-P$ distance $(\mathrm{mm})$ & $P-C$ angle $\left({ }^{\circ}\right)$ \\
\hline 1 & $32.7 / 33.7$ & $35 / 19$ \\
2 & $26.5 / 28.5$ & $32 / 30$ \\
3 & $21.2 / 20.5$ & $29 / 35$ \\
4 & $29.5 / 38.7$ & $27 / 34$ \\
5 & $20.0 / 21.4$ & $23 / 36$ \\
6 & $27.3 / 26.2$ & $27 / 33$ \\
7 & $26.2 / 25.9$ & $29 / 21$ \\
8 & $33.6 / 35.7$ & $25 / 29$ \\
9 & $24.0 / 23.2$ & $20 / 24$ \\
10 & $23.2 / 25.1$ & $21 / 36$ \\
Average & $25.0 / 25.5$ & $26.8 / 29.7$ \\
\hline
\end{tabular}

L-P distance $=$ lumbar fascia - psoas major muscle distance on puncture line; $\mathrm{P}-\mathrm{C}$ angle $=$ puncture point - the posterior colon margin angle. patients had not undergone retroperitoneal procedure previously. All laparoscopic procedures were performed by the retroperitoneal approach.

The time to establish the retroperitoneal cavity was 3 to 6 minutes (mean, 4 minutes). All patients underwent retroperitoneal laparoscopic operation without converting to open procedure. The retroperitoneal space was large enough to perform retroperitoneoscopic procedures for the adrenal gland, kidney, and upper ureter. Of importance, no injury of the intestine, colon, kidney, pleura, peritoneum, large blood vessels, and other adjacent organs occurred during establishment of the retroperitoneal space using this new method. No trocars required suturing or fixation. In addition, carbon dioxide leakage was not observed intraoperatively. Five patients showed subcutaneous emphysema after the operation, which resolved spontaneously in 1 to 2 days. Wound hernia was not noted. The follow-up duration of patients ranged from 3 months to 2 years (Table 2). Patients who underwent retroperitoneal laparoscopic radical nephrectomy did not show tumor recurrence or metastasis. Hydronephrosis in patients with upper ureteral calculus was alleviated. Patients with renal cysts did not show recurrence. 
Table 2. Demographic Characteristics of the Patients Receiving Retroperitoneoscopy Using the Direct Puncture Tunnel Method

\begin{tabular}{lc}
\hline Age (years) & 49 \\
Range & $20-86$ \\
Male/female & $186 / 106$ \\
Retroperitoneoscopic procedures & \\
Radical nephrectomy & 30 \\
Adrenalectomy & 56 \\
Nonfunctional nephrectomy & 35 \\
Nephroureterectomy & 10 \\
Upper ureterolithotomy & 22 \\
Renal cyst decortication & 139 \\
Total & 292 \\
Time of establishing retroperitoneal & 4.0 \\
cavity (minutes) & \\
Range & $3-6$ \\
Complications of direct puncture & \\
tunnel method & \\
Trocar misplacement & $0 \%(0 / 292)$ \\
Visceral injury (intestine, colon, & $0 \%(0 / 292)$ \\
$\quad$ kidney, liver, spleen) & \\
Peritoneal membrane rupture & $0 \%(0 / 292)$ \\
Pleura injury & $0 \%(0 / 292)$ \\
Great vessel injury & $0 \%(0 / 292)$ \\
Carbon dioxide leakage & $0 \%(0 / 292)$ \\
Subcutaneous emphysema & $1.7 \%(5 / 292)$ \\
Wound hernia & $0 \%(0 / 292)$ \\
\hline
\end{tabular}

\section{Discussion}

Retroperitoneal laparoscopy provides direct access to the retroperitoneal organs and avoids the potential complications of transperitoneal laparoscopy. ${ }^{9}$

However, the main difficulties of this method are access and creation of the retroperitoneal space. ${ }^{10}$ The risk of serious bleeding, peritoneal rupture, and peripheral organ damage during cavity construction can adversely affect subsequent procedures, prolonging the operation time, and perhaps even resulting in conversion to open procedure. Furthermore, the risk of surgical complications is increased.

A glove finger secured around a stiff catheter was originally used to establish the retroperitoneal space. ${ }^{8}$ Other devices, such as the balloon trocar sheath and a condom, have also been proposed. As an alternative to the homemade balloon devices, several commercial balloon dilators have recently become available. ${ }^{11,12}$ In these methods, briefly, the retroperitoneal space was established by injecting water or air into the glove or balloon.

Although these approaches have been reported to have a high success rate, ${ }^{13}$ significant disadvantages were also observed in clinical practice. For the glove-finger balloon, the tip cannot be directed into the required position accurately and consistently. Fragments of broken gloves need to be carefully searched for and removed, thus prolonging the operation time. To place the balloon dilator in a suitable position, finger dissection or sharp entry into Gerota's fascia is necessary. However, this has a high likelihood of puncturing Gerota's fascia and even the kidney perirenal fat and is not appropriate for radical nephrectomy. In addition, the second port is also difficult to place. If the second trocar is guided by a finger, injury to the physician is possible. Even under endoscopic visualization, it is difficult to safely place the second port owing to the blind zone within the space. To place the balloon dilator more accurately, the skin and fascia incision needs to be enlarged and water or gas injection of a glove or balloon has to be increased in obese patients, which increases the risk of rupture proportionately. Finally, significant carbon dioxide leakage and trocar repeated exfoliation can occur with a sutured and fixed trocar.

It was reported that the retroperitoneal space could also be created by the open laparoscopic method using a Hasson cannula, with a high success rate. ${ }^{14}$ However, the traditional Hasson cannula is heavy and cumbersome, and in patients with a small body size, it may hinder the placement of other trocars into the narrower space. To prevent air leakage, two securing sutures are needed to fix the lateral wing of the cannula.

Therefore, it is necessary to find a simple and feasible method to establish the retroperitoneal cavity without special instruments.

Capelouto et al. ${ }^{15}$ originally proposed the direct insufflation method. Anatomical and radiographic studies indicated that the peritoneal reflection was consistently anterior to the posterior axillary line. ${ }^{16}$ Moreover, when a patient was placed in the lateral position, the anteroposterior extent of the potential retroperitoneal space increased two-fold. Hence, they proposed that it is safe to insert a 14-gauge Veress needle into the retroperitoneum directly. Chiu et al. ${ }^{17}$ found significant anterior movement of the colon in patients who moved from the supine to lateral position, which provided space for inserting the Veress needle blindly into the retroperitoneum. In our method, we also chose this location as the first puncture point, but we omitted the Veress needle procedure. In addition, the following procedure of our method is also different.

We found that if the patient is in the lateral position and the hips are flexed by the lumbar bridge, the kidneys will naturally fall toward the side of the head because of gravity. The retroperitoneal region between the lower pole of the kidney and upper edge of the pelvic cavity has only adipose connective tissue. This direction has the largest amount of fat in the retroperitoneal space. According the CT scans of the lateral decubitus position, there are no visceral or blood vessels on the first trocar puncture route, and there is a large angle between the colon and the puncture route. Therefore, as long as the first trocar is inserted vertically into the retroperitoneal cavity, it will not damage the visceral or blood vessels. An L-P line over $2 \mathrm{~cm}$ will also cause the first trocar to pass through the lumbodorsal fascia with a noticeable sense of frustration. Thus, it is safe to blindly puncture this region with the trocar. We could see that the first disposable trocar passed through the skin, superficial fascia, muscle layer, and lumbar fascia and entered the retroperitoneal cavity while inserting the laparoscope. The fat and psoas major muscle in the retroperitoneal cavity under monitor could also help us to make sure that the first trocar is in the retroperitoneal space. Of course, it is also necessary to inspect for damage to the visceral or blood vessels while inserting the laparoscope. To prevent organ damage, the placement of the first trocar should be shallow rather than deep. It is necessary to read the CT scans carefully before operation to avoid any anatomical variations especially in patients with spinal deformities. 
Carbon dioxide gas easily diffuses into the loose tissue gap, such as the anterior space of the psoas major muscle. Because of the compactness and integrity of Gerota's fascia, it is difficult to fill this space with carbon dioxide. The fat in front of the psoas major muscle is sparse and avascular; thus, it can be easily distinguished. It is safe to move the laparoscopy head toward the costal ridge angle in this area under the monitor. The psoas major muscle provides anatomical markers for the operation, and the absence of blood vessels in front of the psoas major muscle limits bleeding during the procedure. Therefore, it is safe and feasible for the laparoscopy to tunnel the retroperitoneal space in the anterior space of the psoas major muscle.

When the second trocar is successfully placed, subsequent operations become very easy and simple. In our study, we do not advocate placing the second port posterior to the posterior axillary line. The maneuverability of the instruments inserted through a posteriorly located port is limited because the surgeon must work over the "hump" of the psoas and spinal erector muscles. ${ }^{18}$ An anteriorly placed trocar allows for greater maneuverability. Small vessels are electrocoagulated, fibers can be cut through the second trocar, and the fat and peritoneum are compressed to expand the retroperitoneal space. There is almost no bleeding in the process of establishing the retroperitoneal cavity.

Our method uses the same equipment as that in transperitoneal laparoscopy and does not require additional special equipment. There are several advantages to using our new novel surgical method. The issue of carbon dioxide leakage is eliminated using this technique. Provided the length of the skin incision is determined by the trocar diameter, each trocar does not need to be sutured and fixed. Because they are not used, there is no risk of rupture of gloves or balloons. Except for placement of the first trocar, which is a blind puncture, all other operations are performed under surveillance. Furthermore, in our method, Gerota's fascia is not destroyed in accordance with the principles of renal tumor procedure. The time required for cavity construction is also very short. In clinical application, there was a high success rate and low complication rate using this method. In our case, subcutaneous emphysema was related to the patient's body shape; patients who exhibited subcutaneous emphysema had a slender body shape. Subcutaneous emphysema quickly resolved spontaneously and, thus, was not a major complication.

Our study has some limitations. Children and patients with severe adhesions after previous renal procedure were not enrolled in our study. We plan to determine the effectiveness of this technique in other populations in the future.

\section{Conclusions}

Taken together, our method provides a simple, effective, and safe way to establish the retroperitoneal space in adults.

\section{Authors' Contributions}

J.L. contributed to protocol/project development, data collection and management, data analysis, and article writing/editing. B.L. contributed to protocol/project development data collection and management. J.A. contributed to data collection and management, and article writing/editing. L.Z. contributed to data collection and management. K.W. contributed to protocol/project development.

\section{Ethical Standards}

All procedures performed in studies involving human participants were in accordance with the ethical standards of the Institutional and/or National Research Committee and with the 1964 Declaration of Helsinki and its later amendments or comparable ethical standards.

\section{Author Disclosure Statement}

No competing financial interests exist.

\section{Funding Information}

This work was supported by the Medical Research Project of Sichuan Province, (grant no. Q18040) and a Foundation Project from the Health and Family Planning Committee of Sichuan Province (grant no. 16PI294).

\section{Supplementary Material}

Supplementary Video S1

\section{References}

1. Wittmoser R. Possibilities of using sympathectomy for treatment of pain syndromes. Stereotact. Funct Neurosurg 1984;47:203-207.

2. Fitzpatrick JM, Wickham JE. Minimally invasive surgery. Br J Surg 1990;77:721-722.

3. Kerbl K, Figenshau RS, Clayman RV, et al. Retroperitoneal laparoscopic nephrectomyLaboratory and clinical experience. J Endourol 1993;7:23-26.

4. Gaur DD. Laparoscopic operative retroperitoneoscopy: Use of a new device. J Urol 1992;148:1137-1139.

5. Gill IS, Munch LC, Lucas BA, Das S. Initial experience with retroperitoneoscopic nephroureterectomy: Use of a double-balloon technique. Urology 1995;46:747-750.

6. Gasman D, Saint F, Barthelemy Y, Antiphon P, Chopin D, Abbou CC. Retroperitoneoscopy: A laparoscopic approach for adrenal and renal surgery. Urology 1996;47: 801-806.

7. Rassweiler JJ, Teber D. Advances in laparoscopic surgery in urology. Nat Rev Urol 2016;13:387-399.

8. Rassweiler JJ, Seemann O, Frede T, Henkel TO, Alken P. Retroperitoneoscopy: Experience with 200 cases. J Urol 1998;160:1265-1269.

9. Burghelea C, Ghervan L, Barbos A, et al. Role of retroperitoneal laparoscopic nephroureterectomy in the current treatment of upper urinary tract urothelial carcinoma. Chirurgia (Bucur) 2008;103:435-443.

10. Fan X, Xu K, Lin T, et al. Comparison of transperitoneal and retroperitoneal laparoscopic nephrectomy for renal cell carcinoma: A systematic review and meta-analysis. BJU Int 2013;111:611-621.

11. Rassweiler JJ, Henkel TO, Stoch C, et al. Retroperitoneal laparoscopic nephrectomy and other procedures in the upper retroperitoneum using a balloon dissection technique. Eur Urol 1994;25:229-236.

12. Tai HC, Lai MK, Chueh SC, Chen SC, Hsieh MH, Yu HJ. An alternative access technique under direct vision for preperitoneoscopic pelvic surgery: Easier for the beginners. Ann Surg Oncol 2008;15:2589-2593. 
13. McDougall EM, Clayman RV. Advances in laparoscopic urology, Part I. History and development of procedures. Urology 1994;43:420-426.

14. Gett RM, Joseph MG. A safe technique for the insertion of the Hasson cannula. ANZ J Surg 2004;74:797-798.

15. Capelouto CC, Moore RG, Silverman SG, Kavoussi LR. Retro-peritoneoscopy: Anatomical rationale for direct retroperitoneal access. J Urol 1994;152:2008-2010.

16. Tirkes T, Sandrasegaran K, Patel AA, et al. Peritoneal and retroperitoneal anatomy and its relevance for crosssectional imaging. Radiographics 2012;32:437-451.

17. Chiu AW, Chen KK, Wang JH, Huang WJ, Chang LS. Direct needle insufflation for pneumoretroperitoneum: Anatomic confirmation and clinical experience. Urology 1995;46:432-437.

18. Gill IS, Grune MT, Munch LC. Access technique for retroperitoneoscopy. J Urol 1996;156:1120-1124.
Address correspondence to:

Kunjie Wang, MD

Department and Institute of Urology

West China Hospital

Sichuan University

No. 37 Guo Xue Xiang

Chengdu 610041

Sichuan

China

E-mail: wangkj@scu.edu.cn
Abbreviation Used

$\mathrm{CT}=$ computed tomography 